\title{
BMP-7 enhances SnoN mRNA expression in renal tubular epithelial cells under high-glucose conditions
}

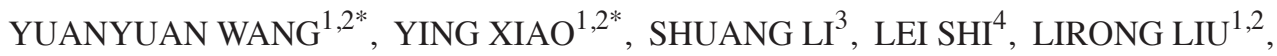 \\ YINGYING ZHANG ${ }^{1,2}$, MINGJUN SHI ${ }^{1,2}$ and BING GUO ${ }^{1,2}$
}

\author{
${ }^{1}$ Department of Pathophysiology; ${ }^{2}$ Laboratory of Pathogenesis Research, Drug Prevention and Treatment \\ of Major Diseases, Guizhou Medical University, Guiyang, Guizhou 550025; ${ }^{3}$ Department of Pathophysiology, \\ Guizhou Medical Hospital, Guiyang, Guizhou 550002; ${ }^{4}$ Department of Pathology, The Second Affiliated Hospital \\ of Guizhou Medical University, Kaili, Guizhou 556000, P.R. China
}

Received December 22, 2016; Accepted July 4, 2017

DOI: $10.3892 / \mathrm{mmr} .2017 .7025$

\begin{abstract}
The present study aimed to identify any association between bone morphogenetic protein-7 (BMP-7) and the expression of the transcriptional co-repressor Ski-related novel protein $\mathrm{N}(\mathrm{SnoN})$, in addition to alterations in tubulointerstitial fibrosis, during the development and progression of diabetic nephropathy (DN). Streptozotocin was injected into the tail veins of 20 healthy and specific pathogen-free male Sprague-Dawley rats. The rats were sacrificed to detect the appropriate biochemical indicators of renal pathological alterations following 24 weeks. Then, various doses of human recombinant BMP-7 were added to high glucose-cultured NRK-52E cells. Immunohistochemistry, immunofluorescence staining and western blotting were used to determine the expression of SnoN, BMP-7, Smad ubiquitin regulatory factor (Smurf)2, Arkadia, E-cadherin, $\alpha$-smooth muscle actin and Collagen III. Reverse transcription-quantitative polymerase chain reaction was used to detect SnoN mRNA expression. With the progression of DN, the expression of BMP-7 in rat renal tissue was downregulated, whereas the expression of Smurf2 and Arkadia increased. Furthermore, the expression of SnoN mRNA increased however the expression of SnoN protein decreased, accompanied by renal tubular epithelial cell mesenchymal transition, extracellular matrix (ECM) deposition and severe renal function disorder. The exogenous recombinant human BMP-7 alleviated high glucose-induced phenotypic transformation and ECM synthesis of NRK-52E in vitro and upregulated SnoN transcription and protein expression, however no effect was observed on the expression
\end{abstract}

Correspondence to: Dr Bing Guo, Department of Pathophysiology, Guizhou Medical University, Dongqing Road, Guiyang, Guizhou 550025, P.R. China

E-mail: guobingbs@126.com

*Contributed equally

Key words: diabetic nephropathy, SnoN, TGF- $\beta 1 /$ Smads signaling pathway, bone morphogenetic protein-7, Smurf2, Arkadia of Smurf2 and Arkadia. BMP-7 may ameliorate DN and renal fibrosis via increasing the expression of SnoN mRNA and protein in renal tubular epithelial cells, rather than directly inhibiting the degradation of SnoN by E3 ubiquitin ligase.

\section{Introduction}

The expression of transforming growth factor- $\beta 1$ (TGF- $\beta 1$ ) is upregulated in the course of multiple renal fibrosis and acts as an important cytokine in the renal fibrosis disease process (1-4). It induces the transition of renal tubular epithelial cells (RTECs) to mesenchymal cells and finally into myofibroblasts, increasing the deposition of extracellular matrix (ECM) $(5,6)$. In recent years, many studies have demonstrated that transcriptional corepressor Ski-related novel protein $\mathrm{N}$ (SnoN), an important negative regulatory factor, inhibits the transcription activation of Smads-mediated TGF-1 target gene $(7,8)$. It is well accepted that the diminution of SnoN protein is involved in the development and progression of renal fibrosis, and the downregulation of SnoN protein facilitates renal fibrotic lesions while the upregulation of which slows down the development of renal fibrosis $(1,8)$.

Studies found that bone morphogenetic protein-7 (BMP-7), a member of TGF- $\beta 1$ superfamily, is highly expressed in kidney and plays a renal protective effect in maintaining the normal development of kidney, reversing epithelial-to-mesenchymal transition (EMT) $(3,9)$, and in restricting the deposition of ECM $(4,10)$, but the exact mechanism is still unclear. Recent studies have shown that BMP-7 may play a role in anti-renal fibrosis by upregulating the protein expression of SnoN, which is subject to regulation strictly in activation of both transcription and protein stability $(4,11,12)$. Therefore, this study aimed to explore the associations between and among BMP-7, the expression of transcriptional corepressor SnoN and the changes of tubulointerstitial fibrosis during the development and progression of diabetic nephropathy (DN). Furthermore, this study also aimed to demonstrate BMP-7 may have effect on the target of anti-fibrotic and potential mechanism by observing its influence on the expression of SnoN and fibrosis of RTECs while exogenous rhBMP-7 was added to RTECs cultured under hyperglycemic conditions. 


\section{Materials and methods}

Animal model. A total of 20 healthy and specific pathogen-free male Sprague-Dawley rats (weight: $180 \pm 20 \mathrm{~g}$ ) were provided by Beijing HFK Bioscience Co., Ltd. (Beijing, China), and housed in the animal center of Guiyang Medical University, (Guizhou, China). The study was conducted in accordance with the guidelines of the National Health and Medical Research Council of China's Code for the care and use of animals for scientific purpose. All rats were randomly divided into diabetic group (DM group, $\mathrm{n}=10$ ) and nomal control group (NC group, $n=10$ ). Diabetic rats were produced by injecting 0.01-mol/1 streptozotocin (STZ, prepared with sterile citric acid-sodium citrate buffer, $\mathrm{pH}$ 4.5; Sigma, St. Louis, MO, USA) in the tail vein at a dose of $55-\mathrm{mg} / \mathrm{kg}$. Fasting blood glucose level of all rats was detected after $48-\mathrm{h}$. The blood glucose level $\geq 16.7-\mathrm{mmol} / 1$ indicates that the diabetic rat model was established successfully. Rats in NC group were age-matched and injected an equal volume of solvent. Rats in each group were given normal diet and unlimited drinking water. After 24-weeks, 24-h urine of each rat was collected in metabolic cage, and the total volume of urine was recorded before rats were sacrificed. The rats were fasted for $6-8 \mathrm{~h}$ before being anesthetized by diethyl ether, and their femoral arteries were punctured to collect blood samples, which were centrifuged at $4^{\circ} \mathrm{C}$ to separate serum. Urine and serum were stored at $-20^{\circ} \mathrm{C}$ for measuring urine protein and biochemical indices. Kidneys of each rats were harvested, one was fixed in $4 \%$ paraformaldehyde for paraffin sections, and the other one was snap-frozen in liquid nitrogen and stored at $-80^{\circ} \mathrm{C}$ for RNA and protein extractions.

Tests of biochemical markers. The oxidase method was used to measure serum glucose, and the Coomassie Brilliant Blue method was used to measure urine protein. All tests were analyzed by 1650 automatic biochemical analyzer (Beckman Instruments, Inc., Brea, CA, USA), according to the manufacturer's instruction. Urine protein excretion $(\mathrm{mg} / 24-\mathrm{h})$ was assessed as follows: urine protein $(\mathrm{mg} / \mathrm{ml}) \mathrm{x}$ urine volume $(\mathrm{ml}) / 24-\mathrm{h}$.

Histopathological analysis. Kidneys were fixed in paraformaldehyde, embedded in paraffin, and cut by transversing transverse sections $(4-\mu \mathrm{m})$ for staining. Renal tissue morphology was observed under a light microscope by hematoxylin-eosin (H\&E) stain, while renal tissue fibrosis was observed by Masson's trichrome and Periodic Acid-Schiff (PAS) stain.

Cell culture and treatments. RTECs (NRK-52E cells) were cultured in Dulbeccos's modified Eagle's medium (Hyclone, Logan, UT, USA) supplemented with $5 \%$ fetal bovine serum (Gibco; Invitrogen, Carlsbad, CA, USA) containing normal glucose $\left(5.5-\mathrm{mmol} / 1\right.$ glucose), seeded in $25-\mathrm{cm}^{2}$ culture flasks, and placed in the incubator with $5 \% \mathrm{CO}_{2}$ at $37^{\circ} \mathrm{C}$. Before changing in a serum-free medium for $20-\mathrm{h}$ to keep the same pace in growth, cells were treated with the following mediums: i) normal-glucose control group (NG group, 5.5-mmol/1 glucose); ii) high-glucose control group (HG group) $(5.5-\mathrm{mmol} / 1$ glucose $+19.5-\mathrm{mmol} / 1$
D-glucose); iii) high glucose +10-ng/ml BMP-7 (HG+10-ng/ml rhBMP-7 group); and (iv) high glucose +20-ng/ml BMP-7 (HG +20-ng/ml rhBMP-7 group). Cells in each group were cultured for 48 -h for further study.

Immunohistochemistry and immunofluorescence stain. The biotin-streptavidin-peroxidase method (ZSBIO, Beijing, China) was used to stain tissue sections which were incubated with different antibodies [rabbit-anti-BMP-7 1:200; rabbit-anti-E-cadherin 1:100; mouse-anti- $\alpha$-SMA 1:100; rabbit-anti-Collagen III (Col-III) 1:150; and rabbit-anti-SnoN 1:200] (all from Santa Cruz Biotechnology, Santa Cruz, CA, USA) to show the distribution and expression of protein in kidney tissues. Phosphate-buffered saline (PBS) was substituted for the primary antibody, and the secondary antibodies used were affinity-purified biotinylated goat anti-rabbit or anti-mouse immunoglobulin $\mathrm{G}$ ( $\mathrm{IgG}$ ). The proteins were visualized by using diaminobenzidine tetrahydrochloride as a chromogen. Tissue sections were counterstained with Mayer's hematoxylin.

Cells cultured on coverslips were washed with PBS twice and fixed with cold methanol: acetone (1:1) for $10 \mathrm{mins}$ on ice. After washing extensively, cells were blocked with bovine serum antigen for $30 \mathrm{mins}$ at room temperature and then incubated with the specific primary antibodies rabbit-anti-cytokeratin-18 (CK-18, 1:100; Biosynthesis, China), rabbit-anti-E-cadherin (1:200), and mouse-anti- $\alpha$-SMA (1:100) overnight at $4^{\circ} \mathrm{C}$. Then cells were stained with fluorescein isothiocyanate (FITC)-conjugated goat-anti-rabbit $\operatorname{IgG}(1: 400)$ or cyanine-3 (Cy3)-conjugated goat-anti-mouse IgG (1:400) (both from Beyotime, Haimen, China). After washing again, the cells were stained with 4',6-diamidino-2-phenylindole (DAPI) to visualize the nuclei, and then observed under an inverted fluorescence microscope. For colocalization of the tubular marker E-cadherin and $\alpha$-SMA on NRK-52E cells, immunofluorescence stain was performed. After being incubated with primary antibodies rabbit-anti-E-cadherin (1:200) at $4^{\circ} \mathrm{C}$ overnight, the slides were then stained with FITC-conjugated goat-anti-rabbit IgG (1:400; Beyotime). After this, the slides were incubated with primary antibodies mouse-anti- $\alpha$-SMA $(1: 100)$ at $4^{\circ} \mathrm{C}$ overnight, and then with Cy3-conjugated goat-anti-mouseIgG (1:400; Beyotime). The nucleus were re-dyed with DAPI. These stained slides were observed under an inverted fluorescence microscope and photographed.

Western blot analysis. The protein expression in renal cortex and cultured cells was analyzed by western blot analysis. Renal tissue and cultured cells were lysed in ice-cold lysis buffer. The protein concentration was determined using the BCA protein assay kit (Beyotime). Samples were separated by sodium dodecyl sulfate-polyacrylamide gel electrophoresis. Then the proteins were electro transferred onto a polyvinylidene difluoride membrane (Millipore, Germany). Non-specific binding to the membrane was blocked in 5\% nonfat milk for $1 \mathrm{~h}$ at room temperature. The membranes were then incubated were different primary antibodies including BMP-7 (1:150), E-cadherin (1:200), $\alpha$-SMA (1:150), Col-III (1:200), SnoN (1:200), Smad ubiquitin regulatory factor 2 (Smurf2; 1:600; Abcam, Cambridge, UK), Arkadia (1:300) 
A

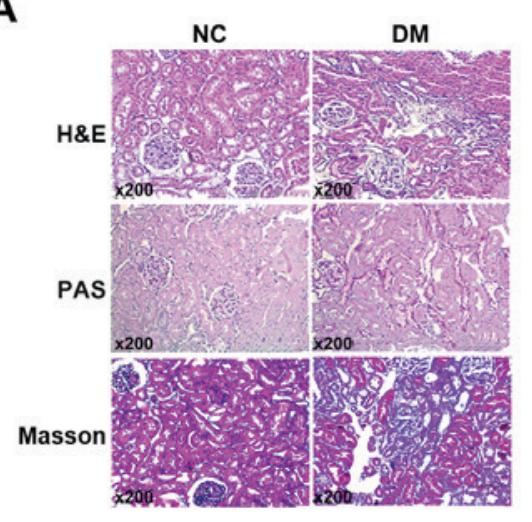

B

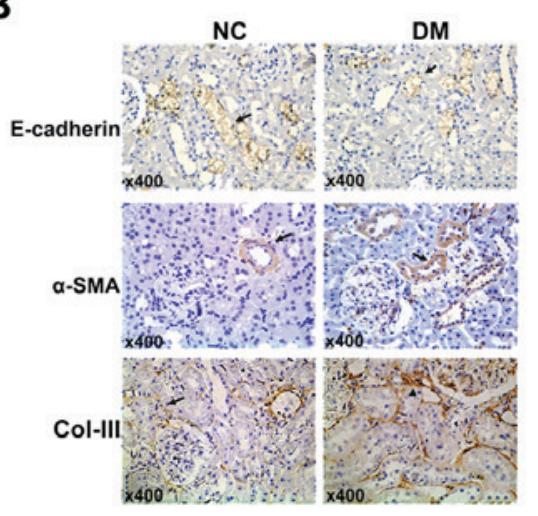

C

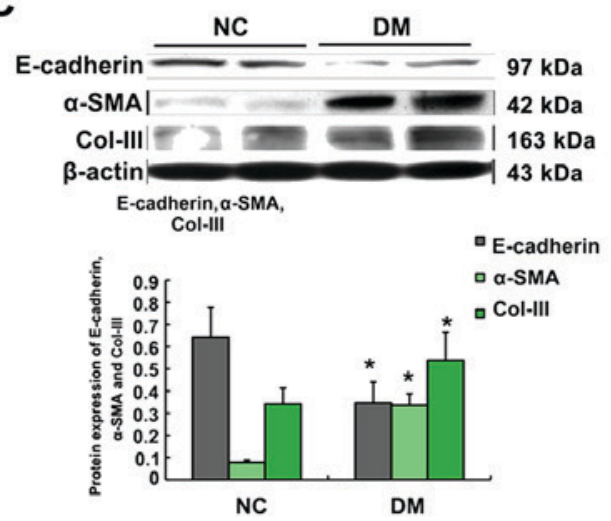

Figure 1. Enhanced renal fibrosis in diabetic rats. Histological changes of kidneys in the NC group and DM group (hematoxylin-eosin staining, Periodic Acid-Schiff staining, and Masson stains, magnification, x200) (A). Immunohistochemical staining of E-cadherin, $\alpha$-SMA, and Col-III in the kidney tissues (magnification, x200). Arrows $(\rightarrow$ ) indicate positive expression (B).Graphical presentations show the protein expression of E-cadherin, $\alpha$-SMA, and Col-III. Mean $\pm \mathrm{SD}, \mathrm{n}=10,{ }^{*} \mathrm{P}<0.05$ vs. $\mathrm{NC}$ group $(\mathrm{C})$.

and $\beta$-actin (1:400) (both from Santa Cruz Biotechnology) for $16 \mathrm{~h}$ at $4^{\circ} \mathrm{C}$. After washing extensively in Tris-buffered saline (TBS), the membranes were incubated with a secondary horseradish peroxidase-conjugated antibody (Santa Cruz Biotechnology) in TBS containing $1 \%$ nonfat milk for $1 \mathrm{~h}$ at room temperature. The membranes were then washed with TBS buffer again. The signals were visualized by using the enhanced chemiluminescence system (Beyotime) and then detected on $\mathrm{x}$-ray films. The Bio-Rad gel imaging system (Bio-Rad, Hercules, CA, USA) was used for image acquisition. The band intensity was quantified by using the Quantity One 4.6 software (Bio-Rad).

Quantitative real-time polymerase chain reaction ( $q R T-P C R$ ). Total RNA in rat renal cortex or NRK-52E cells was extracted by using the TRIzol (Tiangen, Beijing, China) method according to the manufacturer's protocol. First-strand cDNA, which was stored at $-20^{\circ} \mathrm{C}$, was synthesized from $5-\mu \mathrm{g}$ total RNA in renal tissues or 3- $\mu$ g total RNA in NRK-52E cells by using the revert Aid First Strand cDNA Synthesis kit (Fermentas, Lithuania). All quantitative real-time polymerase chain reaction (qRT-PCR) amplification was performed by iQ SYBR-Green SuperMix (Bio-Rad). The primer sequences were as follows: SnoN (ForteBio, Shanghai, China) 5'-CCA TTCAATGCCCCATCCT-3' (sense) and 5'-AGTTCGTGG CCGCAATAAAG-3' (antisense) (the size of the product was $81 \mathrm{bp}$ ); $\beta$-actin (ForteBio) 5'-GCCAACACAGTGCTGTCT-3' (sense) and 5'-AGGAGCAATGATCTTGATCTT-3' (antisense) (the size of the product was $114 \mathrm{bp}$ ). After 45 cycles of amplification, data was collected to plot kinetic curves, and the $\mathrm{Ct}$ value was acquired. The aforementioned steps were repeated for three times, and the $\Delta \Delta \mathrm{Ct}$ method was used to calculate the mRNA/ $\beta$-actin ratio for each group.

Statistical analysis. The SPSS 13.0 software (IBM Corp., Armonk, NY, USA) was used for statistical analysis. All statistics were expressed as mean \pm standard deviation (mean \pm SD). Statistical analysis between the groups was performed using an unpaired Student's t-test, while comparison among multiple groups was performed using one-way analysis of variance followed by Student-Newman-Keuls q-test. Differences were considered significantly at $\mathrm{P}<0.05$.

\section{Results}

Enhanced renal fibrosis in diabetic rats. The symptoms of diabetic rats, which had being injected with STZ, including polydipsia, diuresis, and polyphagia. The levels of 24-h urine protein and blood glucose were significantly higher in diabetic rats than those in normal rats (24-h protein: $305.36 \pm 42.49$ vs. $39.41 \pm 11.31 \mathrm{mg}$; blood glucose: $27.42 \pm 3.930$ vs. $6.84 \pm 1.58 \mathrm{mmol} / \mathrm{l})(\mathrm{P}<0.05)$.

H\&E, PAS, and Masson stain showed an increase of mesangial matrix and thickness of glomerular basement membrane in 24-week diabetic rats. Moreover, part of RTECs were atrophied or lost, and the inflammatory cells increased and infiltrated into wider tubulointerstitium (Fig. 1A).

The immunohistochemical analysis revealed that no positive $\alpha$-SMA expression was detected in renal tubules except around the blood vessels in tubulointerstitium of normal kidneys, but it was remarkable in renal tubules of diabetic rats. Moreover, Col-III deposition was much more confined within the areas of tubulointerstitium in kidneys of diabetic rats when comparing with $\mathrm{NC}$ group. However, epithelial cell marker, E-cadherin, was much lower in the tubular epithelium of DM groups when comparing with NC group (Fig. 1B). Being consistent with the immunohistochemical analysis, the Western blot analysis revealed that the expression of $\alpha$-SMA and Col-III increased, but E-cadherin in the kidneys of DM group decreased when comparing with NC group (Fig. 1C). This observation may indicate that RTECs show the transition from the epithelial cells to the mesenchymal cells.

Effect of diabetes on BMP-7, SnoN, Smurf2, and Arkadia expression in renal tissues of rats. The results of immunohistochemistry showed that, in rat renal tissues, BMP-7 mainly expressed in RTECs. Western blot indicated that the BMP-7 protein expression in the renal cortex of the DM group was lower than that in NC group (Fig. 2A). As shown in Fig. 2B and $\mathrm{C}$, immunohistochemical stain and Western blot analyses 
A
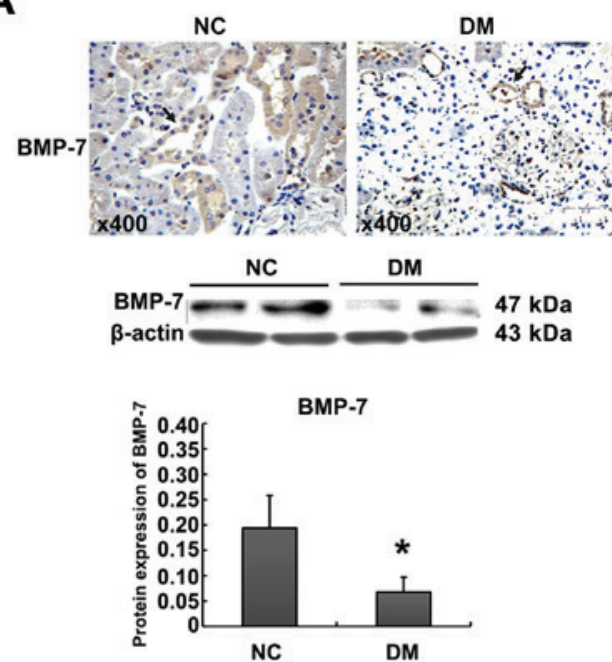

C

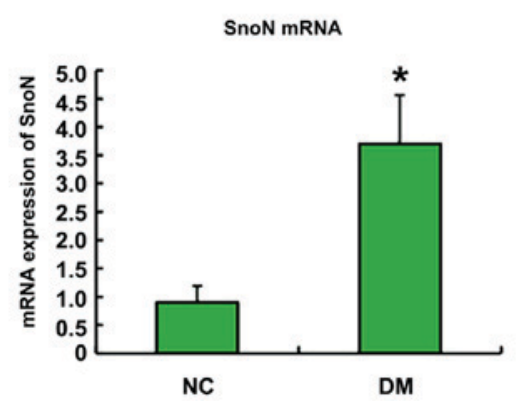

B
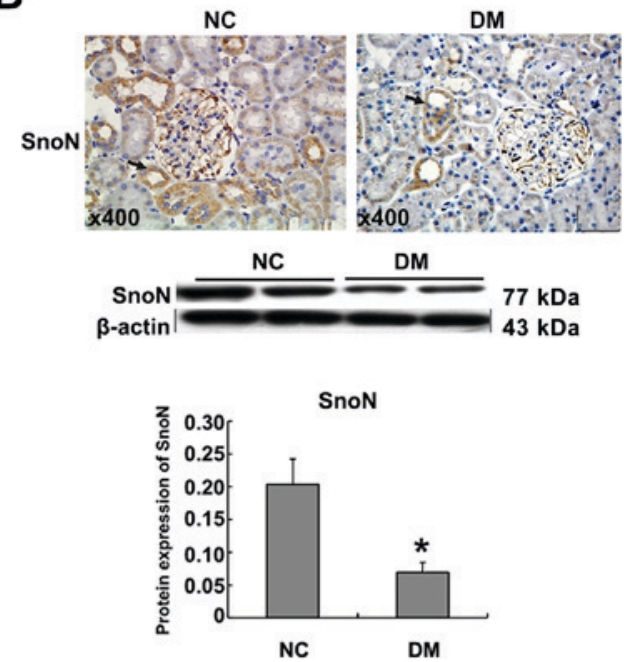

D

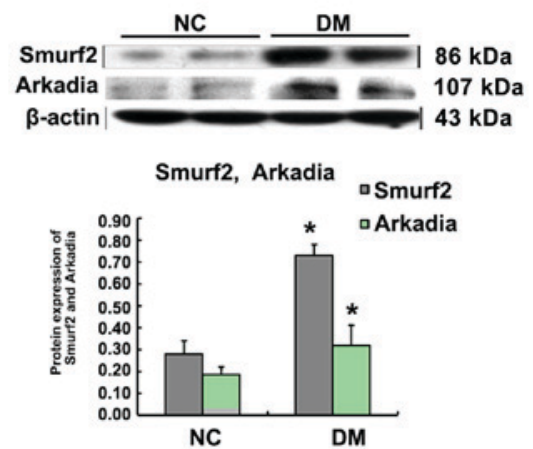

Figure 2. Effect of diabetes on BMP-7, SnoN, Smurf2, and Arkadia expression in the renal tissues of rats. Immunohistochemical staining of BMP-7 in the kidney tissues (magnification, x200). Arrows $(\rightarrow$ ) indicate positive expression. Graphical presentations show the protein expression of BMP-7. Mean \pm SD, $\mathrm{n}=10,{ }^{*} \mathrm{P}<0.05$ vs. NC group (A). Immunohistochemical staining of SnoN in the kidney tissues (magnification, $\mathrm{x} 200$ ). Arrows $(\rightarrow)$ indicate positive expression. Graphical presentations show the protein expression of SnoN. Mean $\pm \mathrm{SD}, \mathrm{n}=10,{ }^{*} \mathrm{P}<0.05$ vs. NC group (B). Graphical presentations show the relative abundance of SnoN mRNA after normalization with $\beta$-actin mRNA. Mean $\pm \mathrm{SD}, \mathrm{n}=10,{ }^{*} \mathrm{P}<0.05$ vs. NC group (C). Graphical presentations show the protein expression of Smurf 2 and Arkadia. Mean $\pm \mathrm{SD}, \mathrm{n}=10,{ }^{*} \mathrm{P}<0.05$ vs. NC group (D).

showed that, in diabetic conditions, the SnoN protein expression was largely lower in vivo when comparing with that in normal rats. In contrast to the significant decrease of SnoN protein, the expression of SnoN mRNA was upregulated in kidneys of diabetic rats when comparing with that in normal rats. The western blot analysis revealed that Smurf2 and Arkadia proteins were weakly expressed in renal extracts from normal rat kidneys. In kidneys of diabetic rats, increased expression of Smurf2 and Arkadia was notable at the protein level (Fig. 2D).

Exogenous rhBMP-7 inhibited the transition from tubular epithelial cells to mesenchymalcells and the expression of Col-III in vitro. The immunofluorescence analysis showed that E-cadherin and CK-18 predominantly localized in the cytomembrane of NRK-52E cells. However, staining for $\alpha-S M A$ was hardly seen in NRK-52E cells (Fig. 3A). These evidences confirmed that the shape and growth of NRK-52E cells were good. As shown in Fig. 3B, E-cadherin progressively diminished in NRK-52E cells cultured in high-glucose medium, when comparing with those cultured in normal-glucose medium for $48 \mathrm{~h}$. However, the $\alpha$-SMA expression increased significantly after high-glucose medium incubation.
Western blot analysis showed that renal fibrosis occurred in the high-glucose group (Fig. 3C). This was demonstrated by a significant upregulation of $\alpha$-SMA and Col-III, and a remarkable downregulation of E-cadherin in NRK-52E cells cultured in high-glucose medium for 48-h. In contrast, these fibrotic changes were largely attenuated in NRK-52E cells co-treated with exogenous rhBMP-7. In high glucose conditions, treating NRK-52E cells with rhBMP-7 suppressed the expression of both $\alpha$-SMA and Col-III, and restored the E-cadherin expression in a dose-dependent manner, peaking at a dose of $20-\mathrm{ng} / \mathrm{ml}$.

Exogenous rhBMP-7 upregulated the expression of SnoN protein by promoting gene transcription independent of Smurf 2 and Arkadia. qRT-PCR demonstrated that the upregulation of SnoN mRNA was inconsistent with the downregulation of SnoN protein as revealed by the Western blot analysis in NRK-52E cells in high-glucose medium when comparing with those in normal-glucose medium (Fig. 4A and B). Moreover, a significant increase in protein expression of Smurf 2 and Arkadia was seen in the cells exposed to high glucose, similar to that in DM group (Fig. 4C). There results from Western blot analysis demonstrated that rhBMP-7 induced SnoN protein 
A

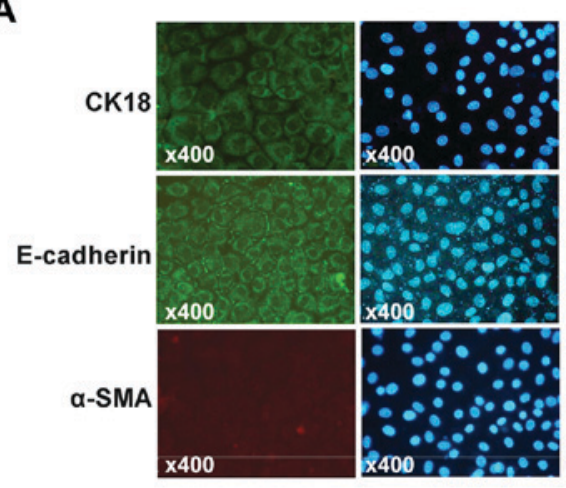

C
B

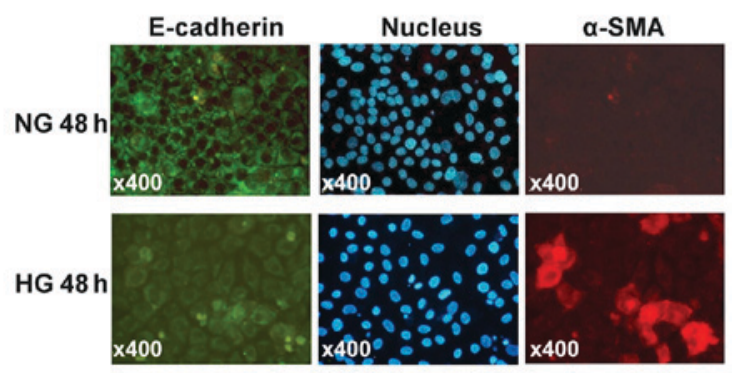

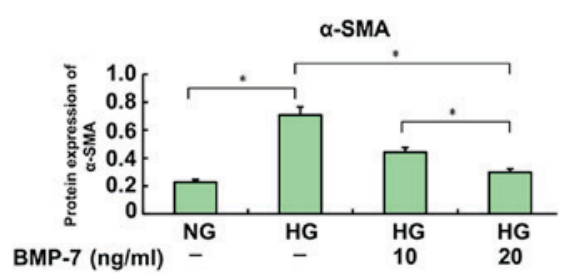
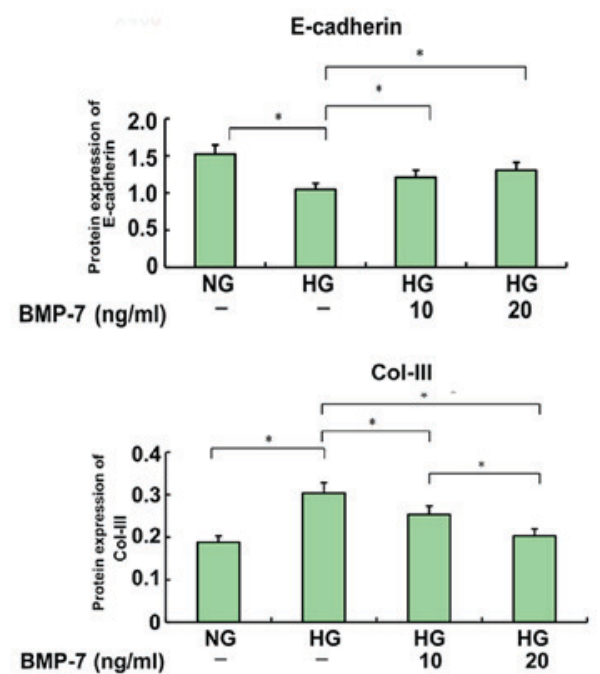

Figure 3. Exogenous BMP-7 inhibited tubular epithelial-to-mesenchymal transition and the expression of Col-III in vitro. Immunofluorescence staining of E-cadherin, $\alpha$-SMA, and CK-18 in NRK-52E cells (A). Immunofluorescence staining of E-cadherin and $\alpha$-SMA in NRK-52E cells after incubation in different conditions. DAPI staining (blue) represents cell nucleus (magnification, $\mathrm{x} 400$ ) (B). Western blotting demonstrated the expression of E-cadherin, $\alpha$-SMA, and Col-III. Graphical presentations show the protein expression of E-cadherin, $\alpha$-SMA, and Col-III. Mean \pm SD, $n=3,{ }^{*} \mathrm{P}<0.05$ (C).

expression in a dose-dependent manner, peaking at $20-\mathrm{ng} / \mathrm{ml}$ dose. In contrast, there was no effect on the expression of Smurf2 and Arkadia proteins in NRK-52E cells which were co-treated with or without exogenous rhBMP-7 exposed to high glucose (Fig. 4C). Further studies by qRT-PCR indicated that gene transcription of SnoN resulted in higher levels of SnoN mRNA in NRK-52E cells co-treated with exogenous BMP-7 when exposure to high glucose (Fig. 4B). All these results strongly suggested that BMP-7 upregulated the SnoN expression in NRK-52E cells.

\section{Discussion}

Numerous studies have suggested that the TGF- $\beta 1 /$ Smads signaling pathway activates $\operatorname{Smad} 2 / 3$, which subsequently combine with Smad4 and translocate into the cell nucleus, and finally control the transcription of TGF- $\beta 1$ gene, exerting its fibrotic effect on progression of DN $(1,6)$. Thus, how to inhibit or block the TGF- $\beta 1 /$ Smads signaling pathway has become the focus of recent research. SnoN was discovered early as an oncoprotein, due to the induction of transdifferentiation in chicken embryo fibroblasts (7). The most important mechanism of SnoN is switching the function of TGF- $\beta 1 /$ Smads signal transduction pathways $(6,7)$. Recently, SnoN was knocked down by using siRNA following high-glucose stimulation, which caused a significant reduction of E-cadherin in primary RTECs, while the upregulation of fibronectin (FN) and $\alpha$-SMA could further increase RTECs fibrosis. On the contrary, the overexpression of SnoN could reduce high glucose-mediated RTECs fibrosis (1). So, the downregulation of SnoN protein may be an important pathogenesis of DN renal fibrosis. In this study, type 1 DM rat model was established by injecting STZ into tail vein. Continuous DM status combined with significant proteinuria and renal histological observation showed significant renal tubulointerstitial fibrosis, were regarded as the model with DN. The expression of SnoN and E-cadherin reduced in renal tissue of DM group rats, while $\alpha$-SMA and Col-III increased significantly in renal tissue. This confirms that the reduction of SnoN proteins could promote the development of DN, which is the same as the preliminary findings of our study (1).

The present study found that the increase in the mRNA level of SnoN was not consistent with the protein expression in DM group. It was found that TGF- $\beta 1$ upregulated the transcription of SnoN by binding directly to sno promoter's SBE (Smad-binding element) through the $\mathrm{p}$-smad2/Smad4 complex. This induced the expression of SnoN mRNA (13), which acted as a negative feedback inhibitor for the TGF- $\beta 1 /$ Smads 
A
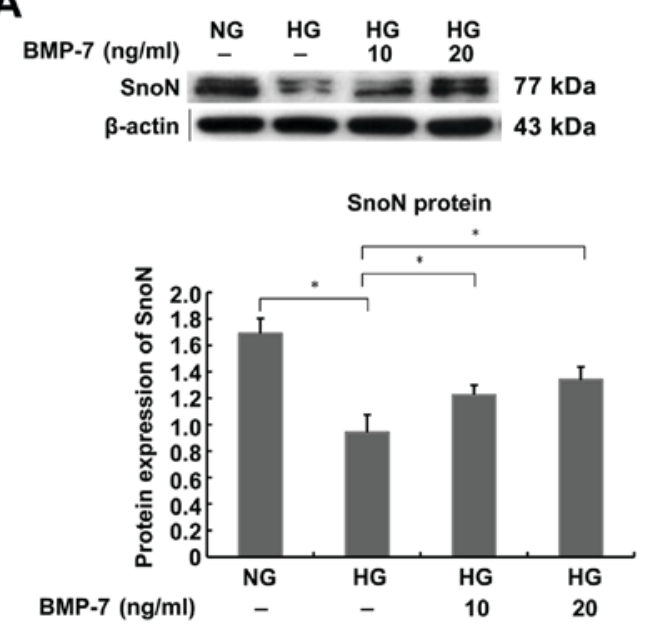

B

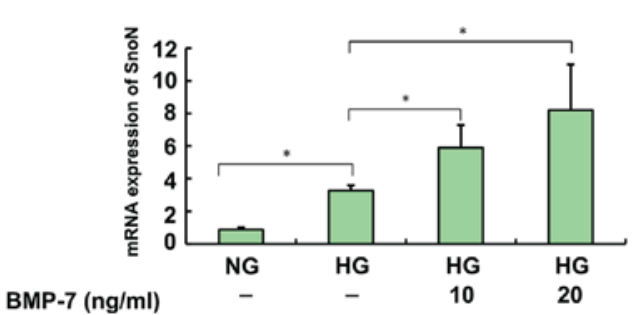

C
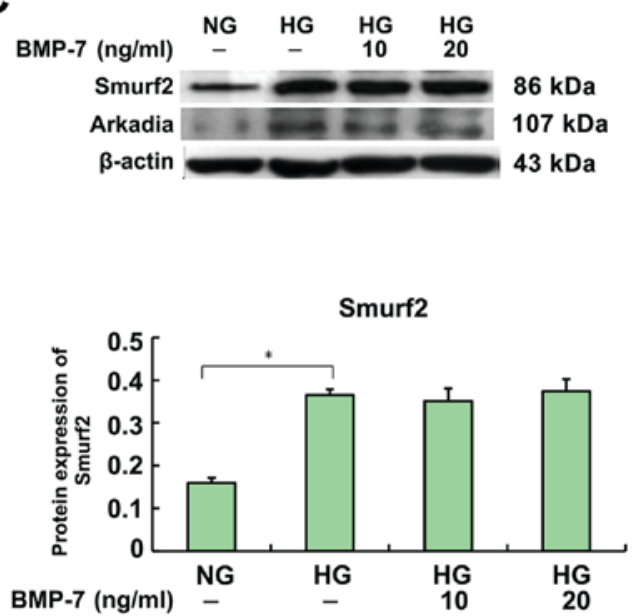

Arkadia

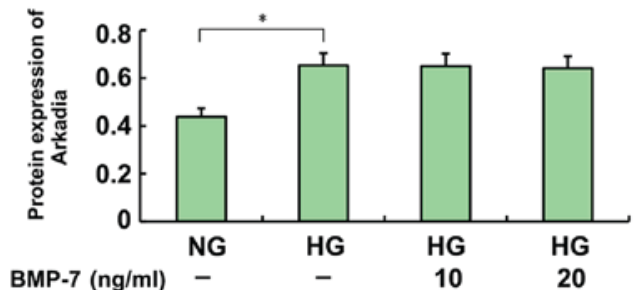

Figure 4. Exogenous BMP-7 upregulated the expression of SnoN protein by promoting gene transcription independent of Smurf2 and Arkadia. Western blotting demonstrated the protein expression of SnoN. Graphical presentations show the protein expression of SnoN. Mean $\pm \mathrm{SD}$, $\mathrm{n}=3$, ${ }^{*} \mathrm{P}<0.059$ (A). Graphical presentations show the relative abundance of SnoN mRNA after normalization with $\beta$-actin $\mathrm{mRNA}$. Mean $\pm \mathrm{SD}$, $\mathrm{n}=3$, ${ }^{*} \mathrm{P}<0.05$ (B). Western blotting demonstrated the expression of Smurf2 and Arkadia. Graphical presentations show the protein expression of $\mathrm{Smurf} 2$ and $\mathrm{Arkadia}$. Mean $\pm \mathrm{SD}, \mathrm{n}=3,{ }^{*} \mathrm{P}<0.05(\mathrm{C})$.

pathway. Another research showed that TGF- $\beta 1$ activated the PI3K/AKT pathway and upregulated the expression of SnoN mRNA (14). Especially, TGF- $\beta 1$ increased the SnoN transcription, strongly inducing the expression of SnoN mRNA in cells and kidney tissues of unilateral ureteral obstruction (UUO) rats (15). However, the level of protein was not coupled with the expression of SnoN mRNA, as TGF- $\beta 1$ protein led to a reduction of SnoN rapidly and significantly. This decrease was mainly connected with Arkadia and Smurf2, an E3 ubiquitin enzyme, which specifically recognized SnoN and involved in activating Smads-mediated ubiquitination and degradation of SnoN (15-17). During DN, high expression of Smurf2 and Arkadia was observed in renal tissue, resulting in the TGF- 31 -mediated interaction of Smad2, SnoN, and Smurf2, leading to the ubiquitin degradation of SnoN protein $(17,18)$. However, TGF- $\beta 1$-activated Smad3 interacted with Arkadia, inducing the degradation of SnoN and enhancing biological effects of p-smad3 $(12,17,19)$. Thus, the expression of SnoN protein facilitates the balance of gene expression and degradation of ubiquitin: The protein level of SnoN, due to degradation by the ubiquitin-proteasome system, reverses the promotion of TGF- $\beta 1 /$ Smads pathway on SnoN transcriptional activation in renal disease processes, which induces fibrosis cascade effects that enhance the DN fibrosis, similar to the report by Fukasawa et al in UUO (15).

BMP-7 has been proved to be anti-fibrotic as it counteracts the TGF- $\beta 1 /$ Smads pathway in many fibrotic diseases including pulmonary fibrosis (20), liver fibrosis (21), and renal fibrosis $(3-5,22)$. However, in a variety of fibrotic diseases, BMP-7 protein downregulation prompts the development of fibrosis. The present study showed that the expression of BMP-7 reduced gradually with the progression of DN. Transfection of recombinant BMP-7 with adenoviral gene or direct addition of rhBMP-7 into RTECs could reduce or counteract the occurrence of EMT and the synthesis of ECM induced by TGF- $\beta 1(9,10,23)$. However, the mechanism of BMP-7 inhibiting the TGF- $\beta 1 / \mathrm{Smad}$ signaling pathway is not clear, only few studies explained the mechanism that BMP-7 does not affect the synthesis of TGF- $\beta 1$ induced by high glucose in RTECs (24). And Luo et al found that $\mathrm{SnoN}$, a negative regulator protein of the TGF- $\beta 1$ signaling pathway, may be involved in the mechanism, and when SnoN was knocked down, the effect of BMP-7 inhibiting the TGF- $\beta 1 /$ Smad signaling pathway was weakened (4). But the specific patterns and mechanisms were not clear. So, in this study, the tubular epithelial cells were cultivated in vitro (NRK-52E cells) in high glucose with various doses of rhBMP-7 (10- and 20-ng/ml) simultaneously, and the effects of rhBMP-7 were observed on the transcription and protein levels of SnoN and the expression of E3 ubiquitin ligases, Smurf2 and Arkadia, to clarify the influence and mechanism of BMP-7 on the expression of SnoN.

BMP-7 has a variety of biological effects, and the doses of rhBMP-7 that are applied for treating fibrotic diseases range from 5- to $400-\mathrm{ng} / \mathrm{ml}$. In the present study, 10- and 20-ng/ml doses of rhBMP-7 were added to high glucose-cultured RTECs to observe whether the fibrosis effect 
is being delayed. The results showed that rhBMP-7 depressed the phenotype transition of RTECs and synthesis of Col-III in a dose-dependent manner. The results suggested that 10 - and 20-ng/ml rhBMP-7 have therapeutic implications. Moreover, although the high dose of glucose (in the high-glucose group) increased the expression of SnoN mRNA and Smurf2 and Arkadia proteins, exogenous rhBMP-7 (in the rhBMP-7 plus high-glucose group) further enhanced the level of SnoN mRNA significantly in NRK-52E cells under high glucose. And no significant difference was found between the expression of Smurf2 and Arkadia in the rhBMP-7 plus high-glucose group compared with the high-glucose group. In other words, BMP-7 could not inhibit the expression of Smurf2 and Arkadia, but upregulated SnoN at the transcriptional level significantly and even reversed part of the degraded protein, eventually restoring the expression of $\mathrm{SnoN}$ protein to block the TGF- $\beta 1 /$ Smads signal pathway.

In conclusion, the results showed that BMP-7 increased the expression of SnoN protein to inhibit the TGF- $\beta 1 /$ Smads signaling pathway and delay the process of DN. And the effect of BMP-7 in upregulating the expression of SnoN protein is not mitigating E3 ubiquitin ligase protein-induced SnoN protein degradation, but enhancing the SnoN mRNA expression. The mechanism of BMP-7 regulating the expression of SnoN still requires further study.

\section{Acknowledgements}

This research is funded by the National Natural Science Foundation of China (no. 81160094 and 81541102), the Guizhou Province Science and Technology Foundation [no. Guizhou Branch J (2011) 2120], and the Guiyang medical school graduate student education innovation plan special funds (contract no. B201103). We thank Professor Limin Lu of Fudan University for kindly gifting the NRK-52E cells.

\section{References}

1. Liu R, Wang Y, Xiao Y, Shi M, Zhang G and Guo B: SnoN as a key regulator of the high glucose-induced epithelial-mesenchymal transition in cells of the proximal tubule. Kidney Blood Press Res 35: 517-528, 2012.

2. Wang YY, Liu RX, Guo B, Xiao Y, Shi MJ, Pi MJ, Wen QY and Zhang GZ: Down-regulation of PTEN expression in kidney and its role in development of diabetic nephropathy in rats. Sheng $\mathrm{Li}$ Xue Bao 63: 325-332, 2011 (In Chinese).

3. Wang Z, Zhao J, Zhang J, Wei J, Zhang J and Huang Y: Protective effect of BMP-7 against aristolochic acid-induced renal tubular epithelial cell injury. Toxicol Lett 198: 348-357, 2010.

4. Luo DD, Phillips A and Fraser D: Bone morphogenetic protein-7 inhibits proximal tubular epithelial cell Smad3 signaling via increased SnoN expression. Am J Pathol 176: 1139-1147, 2010.

5. Wang S, de Caestecker M, Kopp J, Mitu G, Lapage J and Hirschberg R: Renal bone morphogenetic protein-7 protects against diabetic nephropathy. J Am Soc Nephrol 17: 2504-2512, 2006.

6. López-Hernández FJ and López-Novoa JM: Role of TGF- $\beta$ in chronic kidney disease: An integration of tubular, glomerular and vascular effects. Cell Tissue Res 347: 141-154, 2012.
7. Deheuninck J and Luo K: Ski and SnoN, potent negative regulators of TGF-beta signaling. Cell Res 19: 47-57, 2009.

8. Liu S, Yu N, Zhang XL, Chen XQ and Tang LQ: Regulatory effect of berberine on unbalanced expressions of renal tissue TGF-beta1/SnoN and smad signaling pathway in rats with early diabetic nephropathy. Zhongguo Zhong Yao Za Zhi 37: 3604-3610, 2012 (In Chinese).

9. Xu YF, Wan JX and Jiang DW: Effects of bone morphogenic protein-7 on transdifferentiation and the expression of connective tissue growth factor of human renal tubular epithelial cells induced by transforming growth factor-beta1. Zhonghua Yi Xue Za Zhi 89: 1639-1644, 2009 (In Chinese).

10. Xu Y, Wan J, Jiang D and Wu X: BMP-7 counteracts TGF-beta1-induced epithelial-to-mesenchymal transition in human renal proximal tubular epithelial cells. J Nephrol 22: 403-410, 2009.

11. Jahchan NS and Luo K: SnoN in mammalian development, function and diseases. Curr Opin Pharmacol 10: 670-675, 2010.

12. Tan R, Zhang J, Tan X, Zhang X, Yang J and Liu Y: Downregulation of SnoN expression in obstructive nephropathy is mediated by an enhanced ubiquitin-dependent degradation. J Am Soc Nephrol 17: 2781-2791, 2006.

13. Zhu Q, Pearson-White S and Luo K: Requirement for the SnoN oncoprotein in transforming growth factor beta-induced oncogenic transformation of fibroblast cells. Mol Cell Biol 25: 10731-10744, 2005.

14. Nanjundan M, Cheng KW, Zhang F, Lahad J, Kuo WL, Schmandt R, Smith-McCune K, Fishman D, Gray JW and Mills GB: Overexpression of SnoN/SkiL, amplified at the 3q26.2 locus, in ovarian cancers: A role in ovarian pathogenesis. Mol Oncol 2: 164-181, 2008.

15. Fukasawa H, Yamamoto T, Togawa A, Ohashi N, Fujigaki Y, Oda T, Uchida C, Kitagawa K, Hattori T, Suzuki S, et al: Ubiquitin-dependent degradation of SnoN and Ski is increased in renal fibrosis induced by obstructive injury. Kidney Int 69: 1733-1740, 2006.

16. Miyazono K and Koinuma D: Arkadia-beyond the TGF- $\beta$ pathway. J Biochem 149: 1-3, 2011.

17. Sakairi T, Hiromura K, Takahashi S, Hamatani H, Takeuchi S, Tomioka M, Maeshima A, Kuroiwa T and Nojima Y: Effects of proteasome inhibitors on rat renal fibrosis in vitro and in vivo. Nephrology (Carlton) 16: 76-86, 2011.

18. Bonni S, Wang HR, Causing CG, Kavsak P, Stroschein SL, Luo K and Wrana JL: TGF-beta induces assembly of a Smad2-Smurf2 ubiquitin ligase complex that targets SnoN for degradation. Nat Cell Biol 3: 587-595, 2001.

19. Nagano Y, Mavrakis KJ, Lee KL, Fujii T, Koinuma D, Sase H, Yuki K, Isogaya K, Saitoh M, Imamura T, et al: Arkadia induces degradation of SnoN and c-Ski to enhance transforming growth factor-beta signaling. J Biol Chem 282: 20492-20501, 2007.

20. Yang G, Zhu Z, Wang Y, Gao A, Niu P and Tian L: Bone morphogenetic protein-7 inhibits silica-induced pulmonary fibrosis in rats. Toxicol Lett 220: 103-108, 2013.

21. Wang SL, Yang CQ, Qi XL, Yuan M, Chang YZ, Yang L and Gao HJ: Inhibitory effect of bone morphogenetic protein-7 on hepatic fibrosis in rats. Int J Clin Exp Pathol 6: 897-903, 2013.

22. Zeisberg M, Bottiglio C, Kumar N, Maeshima Y, Strutz F, Muller GA and Kalluri R: Bone morphogenic protein-7 inhibits progression of chronic renal fibrosis associated with two genetic mouse models. Am J Physiol Renal Physiol 285: F1060-F1067, 2003.

23. Yang SW, Wang W, Xu ZC and Zhu YY: Construction of recombinant adenovirus containing BMP-7 gene and its expression in proximal tubule epithelial cells. Zhejiang Da Xue Xue Bao Yi Xue Ban 39: 71-78, 2010 (In Chinese).

24. Wang SN, Lapage J and Hirschberg R: Loss of tubular bone morphogenetic protein-7 in diabetic nephropathy. J Am Soc Nephrol 12: 2392-2399, 2001. 